Pacific Journal of Mathematics

STRONG CYCLIC, PARABOLIC AND CONICAL 


\title{
STRONG CYCLIC, PARABOLIC AND CONICAL DIFFERENTIABILITY
}

\author{
N. D. Lane AND K. D. Singh
}

An arc $A$ in the real inversive plane is strongly conformally differentiable at a point $p$ of $A$ if the circle through a fixed point different from $p$ and two variable points of $A$ converges as these points tend to $p$, and the circle through three points of $A$ which tend to $p$ converges. Analogous definitions of strong parabolic differentiability in the real affine plane, using parabolas and three conditions, and of strong conical differentiability in the real projective plane, using conics and four conditions, may be formulated.

Relations among the strong differentiability conditions in each of the above three geometries can be summarized as follows:

Let $N=I I$ in the inversive case; $N=I I$ or III in the affine case; and $N=I I$, III or IV in the projective case. Then the $N$ th strong differentiability condition implies the $K$ th strong condition for $0<K<N-1$, but it does not imply the $(N-1)$-th strong condition. The $(N-1)$-th strong condition will also hold in each of the three geometries, however, if, in addition to the Nth strong condition, any one of the following is assumed:

(i) The limit characteristic curve of the Nth strong condition is nondegenerate,

(ii) The point $p$ is an end-point of the arc $A$,

(iii) The arc $A$ satisfies a weaker condition $(N-1)$ at $p$.

These results are related to a general principle due to 0 . Haupt which states that if an arc has order $n$ with respect to an $n$-parametric family of characteristic curves, then each point of the arc is $(n-2)$ times strongly differentiable with respect to these curves. The relationship appears in the following manner.

Suppose that a curve of an $n$-parametric family of characteristics is determined by $n$ points of an arc and converges to a curve $C$ of the family as the $n$ points converge to $p$. If $R \notin C$, then no curve of the family through a point $R$ will meet $A$ near $p$ more than $n-1$ times.

2. Notation. An arc $\mathrm{A}$ in the real inversive, affine, or projective plane is the continuous image of a real parameter interval. The letters $P, Q, \cdots$ denote points in the plane; $p, q, \cdots$ denote points of arcs. A neighborhood of $p$ on $A$ is the image of a neighborhood of the parameter $p$ on the parameter interval. Since the points of the arcs which we shall consider will be in one-to-one correspondence with the points of the parameter interval in a small neighborhood of $p$, there will be little danger of ambiguity if the same letters $p, q, \cdots$ denote 
both the points of the parameter interval and their images on $A$.

Whenever several points tend to $p$ on $A$, they may be assumed to be distinct unless otherwise stated.

$C$ will denote a circle, $\pi$ a parabola, and $\gamma$ a conic. The region lying to the left [right] of an oriented line $\mathcal{Q}$ or a circle $C$ is denoted by $\mathfrak{L}_{*}\left[\mathbb{2}^{*}\right]$ and $C_{*}\left[C^{*}\right]$ respectively.

$\pi_{*}$ and $\gamma_{*}$ denote the interiors of the parabola $\pi$ and the conic $\gamma$ respectively, while $\pi^{*}$ and $\gamma^{*}$ denote their exteriors.

The $N$-th strong differentiability condition in the conformal, affine and projective cases will be denoted by $C N^{\prime}, A N^{\prime}$ and $P N^{\prime}$ respectively.

3. Conditions $\mathbf{I}$ and $\mathrm{I}^{\prime}$. Let $A$ be an arc in the real inversive plane.

Condition CI'. There exists a point $R \neq p$ such that if the parameters $s$ and $t$ are sufficiently close to the parameter $p, s \neq t$, then the circle $C(s, t, R)$ through the points $s, t$ and $R$ exists. It converges if $s$ and $t$ tend to $p$.

The special case of Condition $\mathrm{CI}^{\prime}$ in which $s=p$ is called Condition CI; cf. [7,5]. The limit tangent circle of CI is denoted by $C\left(p^{2}, R\right)$. If Condition CI or $\mathrm{CI}^{\prime}$ holds for one point $R \neq p$, then it holds for every point $Q \neq p$. Condition $\mathrm{CI}^{\prime}$ implies

$$
\lim _{\substack{s, t \rightarrow p \\ Q \rightarrow R}} C(s, t, Q)=C\left(p^{2}, R\right) \text { for all } R \neq p .
$$

If we designate $R$ as the infinite point, then Conditions CI and $\mathrm{CI}^{\prime}$ become the Affine or Projective Conditions I and I' respectively.

CondiTION $\mathrm{AI}^{\prime}=$ CoNDITION $\mathrm{PI}^{\prime}$. If the parameters $s$ and $t$ are sufficiently close to the parameter $p, s \neq t$, then the straight line $\mathfrak{L}(s, t)$ through the points $s$ and $t$ exists. It converges if $s$ and $t$ tend to $p$.

Condition $\mathrm{AI}=$ Condition $\mathrm{PI}$ is the special case of this condition when $s=p$.

The following properties are well known.

LEMma 1. A convex arc always satisfies Conditions $\mathrm{AI}$ and $\mathrm{AI}^{\prime}$ at an endpoint; cf. [2, 2.7].

Lemma 2. A convex arc which satisfies Condition $\mathrm{AI}$ at an interior point $p$ also satisfies $\mathrm{AI}^{\prime}$ at $p$; cf. [2, 3.1].

LEMMA 3. If an arc which has no cusp at p, satisfies Condition $\mathrm{AI}$ at $p$, and $\mathrm{AI}^{\prime}$ one-sidedly at $p$, then it also satisfies Condition $\mathrm{AI}^{\prime}$ at $p$. 


\section{Strong cyclic differentiability.}

Condition CII. The are $A$ satisfies CI at $p$ and

$$
C\left(p^{3}\right)=\lim _{\substack{s \rightarrow p \\ s \neq p}} C\left(p^{2}, s\right) \text { exists. }
$$

Condition CII'. $\lim _{t, u, v \rightarrow p} C(t, u, v)$ exists.

The following example shows that $\mathrm{CII}^{\prime}$ does not imply $\mathrm{CI}$ or $\mathrm{CI}^{\prime}$.

The arc $A$ defined by $x=t^{2}, y=t^{3}, 0 \leqq t \leqq 1 / 2 ; x=-t^{3}, y=t^{2}$, $-1 / 2 \leqq t<0$, satisfies $\mathrm{CII}^{\prime}$ at the origin $p$, and

$$
\lim _{t, u, v \rightarrow p} C(t, u, v)=p .
$$

If $R \neq p, \lim _{t, u \rightarrow p} C(t, u, R)$ exists and touches the $x$-axis at the origin when $t>0, u>0$. This limit also exists when $t<0, u<0$, but it then touches the $y$-axis at the origin. Hence Conditions $\mathrm{CI}$ and $\mathrm{CI}^{\prime}$ do not hold at $p$.

Theorem 1. Let $A$ satisfy Condition $\mathrm{CII}^{\prime}$ at $p$. Then $A$ will satisfy $\mathrm{CI}^{\prime}$ at $p$ if any one of the following also holds.

(i) $C=\lim _{t, u, v \rightarrow p} C(t, u, v) \neq p$;

(ii) $p$ is an end-point of $A$;

(iii) A satisfies CI at $p$.

Proof. Assume that $A$ satisfies $\mathrm{CII}^{\prime}$ at $p$.

(i) Assume, for the moment, that $C \neq p$. Choose a point $S$ on $C(t, u, v)$ such that $S$ does not converge to $p$ as $t, u, v$ converge on $A$ to $p$.

Let $R$ be a given point, $R \notin C$, and let $D$ be any accumulation circule of the $C(t, u, R)$. Then the angle between $C(t, u, R)$ and $C(t, u, S)=C(t, u, v)$ may be defined as the amplitude of the cross ratio of $t, R, u, S$. Now this angle tends to 0 as $t, u, v$ tend to $p$. Hence $D$ is the unique circle through $R$ which is tangent to $C$ at $p$. Thus $A$ satisfies $\mathrm{CI}^{\prime}$ at $p$.

Next, preparing for (ii) and (iii), we remove the restriction $C \neq p$. Since $R \notin C$, we can choose a neighborhood $B$ of $p$ on $A$ such that $R \notin C(t, u, v)$ for every choice of $t, u, v$ on $B$. This implies that $C(t, u, R)$ meets $B$ only at $t$ and $u$. If we designate $R$ as the infinite point, this means that $B$ is convex.

(ii) If $p$ is an end-point of $B$, then Lemma 1 implies that $B$ satisfies $\mathrm{AI}^{\prime}$ at $p$.

(iii) If $A$ satisfies CI at $p$, then $C\left(p^{2}, R\right)$ exists and, with $R=\infty$, it is the tangent line of $B$ at $p$. Thus $B$ satisfies AI at $p$. By 
Lemma 2, $B$ also satisfies $\mathrm{AI}^{\prime}$ at $p$.

Thus in both of the cases (ii) and (iii), $C(t, u, R)$ converges as $t$ and $u$ converge on $A$ to $p$. Hence $A$ satisfies Condition $\mathrm{CI}^{\prime}$ at $p$.

\section{Strong parabolic differentiability.}

5.1. Let $A$ be an arc in the real affine plane. If $A$ I holds at $p$ we shall denote the family of nondegenerate parabolas which touch the tangent line $\mathfrak{I}$ of $A$ at $p$ by $\tau$ and its compactification by $\bar{\tau}$. If $Q$ and $R$ are distict points which lie on the same side of $\mathfrak{I}$ and $p, Q, R$ are not collinear, then the quadrangle $p, s, Q, R$ will be convex when $s$ is close to $p$ on $A$. Hence there will be two parabolas through these four points. When $s$ tends to $p$, any limit parabola will be one of the two parabolas of $\bar{\tau}$ which pass through $Q$ and $R$. We call it a tangent parabola of $A$ at $p$; cf. [1, 3].

Condition AII. Let $A$ satisfy AI at $p$ and let the points of $A-\{p\}$ lie in one of the closed half-planes bounded by the tangent $\mathfrak{I}$, say in $\mathfrak{I}_{*} \cup \mathfrak{T}$. If $R \in \mathfrak{I}_{*}$, then the two tangent parabolas of $A$ at $p$ through $R$ and $t$ converge when $t$ tends to $p$; cf. [1, 4.1].

The limit osculating parabolas of $A$ at $p$ through $R$ are denoted by $\pi_{i}\left(p^{3}, R\right), \quad i=1,2$. The family of all the osculating parabolas of $A$ at $p$ is denoted by $\sigma$. The set $\sigma$ is one of the following three subsets of the family $\bar{\tau}$.

Type 1. $\sigma$ is a one-parameter family of parabolas of $\tau$ each of which intersects a given member at $p$ and at exactly one other point.

Type 2. $\sigma$ consists of all the double rays of $\bar{\tau}$ with the common vertex $p$ which lie in $\mathfrak{I}_{*} \cup p$.

Type 3. $\sigma$ consists of all the pairs of parallel lines of $\bar{\tau}$ which lie in $\mathfrak{I}_{*} \cup \mathfrak{T}$; cf. $[1,4.4]$.

5.2. Let $A$ be a convex arc. If $p$ is an interior point of $A$ let $\mathfrak{I}$ and $\mathfrak{I}^{\prime}$ be the one-sided tangents of $A$ at $p$. Let $A-\{p\} \in \mathfrak{I}_{*} \cap \mathfrak{I}_{*}^{\prime}$ Choose a point $R \in \mathfrak{I}_{*} \cap \mathfrak{I}_{*}^{\prime}$. If $p$ is an end-point of $A$, let $A-\{p\}$ and $R$ lie in $\mathfrak{I}_{*}$. Let $t, u, v$, be mutually distinct points which lie sufficiently close to $p$ on $A$. Then there will be two parabolas through $t, u, v, R$. We denote them by $\pi_{1}(t, u, v, R)$ and $\pi_{2}(t, u, v, R)$ cf. [2, 2.8].

5.3. Condition AII'. There exists a point $R \neq p$ with the following properties. If $t, u, v$ are mutually distinct and lie sufficiently close to $p$, then the parabolas $\pi_{i}(t, u, v, R)$ exist, $i=1,2$. They converges as $t, u, v$ converge to $p$. 
We note that $\mathrm{AII}^{\prime}$ does not imply $\mathrm{AI}^{\prime}$. For example the arc $A$ which was discussed in $\S 4$ also satisfies AII' at $p$. In particular, if $R$ lies in the first quadrant, then both of parabolas $\pi_{i}(t, u, v, R)$ tend to the double ray through $R$ with the origin $p$ as the initial point as $t, u, v$ tend to $p$. This arc, however, does not satisfy $\mathrm{AI}$ or $\mathrm{AI}^{\prime}$ at $p$.

If $\mathrm{AII}^{\prime}$ holds, then $\pi_{i}(t, u, v, R)$ always exist when $t, u, v$ are sufficiently close to $p$. Hence a sufficiently small neighborhood of $p$ on $A$ is convex.

We also note the following extension of AII'.

Lemma 4. Let $A$ satisfy AII' at $p$. Then there exists a point $R \neq p$ such that

$$
\lim _{\substack{t, u, v \rightarrow p \\ Q \rightarrow R}} \pi_{i}(t, u, v, Q) \quad \text { exists, } i=1,2 .
$$

A proof is given in [2, 3.3].

LemMa 5. AII' and AI imply AII.

Proof. Let $p$ be an interior point of $A$. Since $A$ is convex, the $\pi_{i}(t, u, v, R)$ are defined when $t, u, v$ are close to $p$ and $R$ and $A-\{p\}$ will lie on the same side of the tangent $\mathfrak{I}$ of $A$ at $p$. Hence the $\pi_{i}\left(p^{2}, v, R\right)$ are defined if $v$ is close to $p$. We can approximate each $\pi_{i}\left(p^{2}, v, R\right)$ by a $\pi_{i}(p, u, v, R)$ with $u$ between $p$ and $v$. Since $\mathrm{A} \mathrm{II}^{\prime}$ holds at $p, \lim _{u, v \rightarrow p} \pi_{i}(p, u, v, R)$ exists. Hence $\lim _{v \rightarrow p} \pi_{i}\left(p^{2}, v, R\right)=$ $\lim _{u, v \rightarrow p} \pi_{i}(p, u, v, R)$ also exists. By [1, Theorem 3], AII also holds at $p$.

We wish to prove

Theorem 2. Let A satisfy $\mathrm{AII}^{\prime}$ at $p$. Then $A$ will also satisfy $\mathrm{AI}^{\prime}$ at $p$ if any one of the following conditions also holds.

(i) $\pi_{i}=\lim _{t, u, v \rightarrow p} \pi_{i}(t, u, v, R)$ is not a double ray through $R$ with the vertex $p$.

(ii) $p$ is an end-point of $A$.

(iii) A satisfies AI at $p$.

Proof. Let $A$ satisfy $\mathrm{AII}^{\prime}$ at $p$. Then there exists a neighborhood of $p$ on $A$ which is convex.

Parts (ii) and (iii) now follow directly from Lemmas 1 and 2 respectively.

We now prepare for (i). If $p$ is an interior point of $A$, then $A$ will satisfy Condition I' one-sidedly at $p$. Since A satisfies AI and AII' one-sidedly at $p$, it will also satisfy AII one-sidedly there; cf. Lemmas 5. As a special case of $\mathrm{AII}^{\prime}, \lim \pi_{i}\left(p^{2}, v, R\right)=\pi_{i}$ and hence $\pi_{i}$ will be a nondegenerate parabola (Type 1), or a double ray with the vertex $p$ (Type 2), or a pair of parallel lines (Type 3).

(i) Assume that $\pi_{i}$ is not a double ray. Thus $\pi_{i}$ is nondegenerate 
or a pair of parallel lines. In either case, both of the one-sided tangents of $A$ at $p$ will be tangents of $\pi_{i}$ at $p$. Hence these one-sided tangents will coincide. By Lemma $2, A$ will satisfy $\mathrm{AI}^{\prime}$ at $p$.

REMARK. The implications (ii), (iv) and (vi) in [2, 3.2] are false and (iii) should read: $A-\{p\}$ and $R$ will lie on the same side of either of the one-sided tangents of $A$.

5.4. Let $A$ be a convex arc and let $t, u, v, w$ be mutually distinct points which lie sufficiently close to $p$ on $A$ in that order. Then there will be two parabolas through these four points. We denote by $\pi_{1}(t, u, v, w)$ that parabola such that the order of the points $t, u, v, w$ on the parabola is the same as their order on A. $\pi_{2}(t, u, v, w)$ denotes the other parabola through these points; ef $[2,2.8]$. We shall be concerned mainly with $\pi_{1}$ 's from now on. It will be convenient to write $\pi(t, u, v w)$ for $\pi_{1}(t, u, v, w)$ and refer to it as the principal parabola through $t, u, v, w$. As a limiting case, $\pi_{1}\left(p^{3}, t\right)$ will be denoted by $\pi\left(p^{3}, t\right)$.

Condition AIII. A satisfies Condition AII at $p$ and $\lim _{t \rightarrow p} \pi\left(p^{3} ; t\right)$ exists.

The limit superosculating parabola is denoted by $\pi\left(p^{4}\right)$. If $p$ is of Type 1 , it is either nondegenerate, or a double ray on $\mathfrak{I}$ with the vertex $p$. If $p$ is an end-point of $A, p$ is of Type $1 b$ [Type 1c] if this double ray is equal [opposite] to the limit of the double ray through $t$ with the vertex $p$ as $t$ tends to $p$ on $A$.

Condition AIII'. Suppose that the points $t, u, v, w$ are mutually distinct and lie on $A$ in that order. If they are sufficiently close to $p$, the parabola $\pi(t, u, v, w)$ exists. It converges as these four points converge to $p$.

Condition AIII' does not, in general, imply AII'. Consider, for example, the arc $A=A \cup p_{4} \cup A_{4}^{\prime}$, where $p$ is the origin, $A_{4}$ is given by

$$
x=s^{2}, y=s^{4}-s^{5}, 0<s<\delta \quad(\text { Type } 1 b)
$$

and $A_{4}^{\prime}$ is given by

$$
x=-s^{2} / 2, y=s^{4}+s^{5}, 0<s<\delta \quad \text { (Type 1c). }
$$

Here $\delta$ is sufficiently small. The arc $A$ satisfies $\mathrm{AI}^{\prime}$ at $p$ and the tangent $\mathfrak{I}$ at $p$ lies along the $x$-axis. Condition AII, however, is not satisfied, since the osculating parabolas of $A_{4}$ at $p$ are given by $(y-\lambda x)^{2}=\lambda^{2} y$, while those of $A_{4}^{\prime}$ at $p$ are given by $(y-\lambda x)^{2}=\lambda^{2} y / 4$. 
By Lemma 5, AII' is not satisfied either. It may be verified that $\pi(t, u, v, w)$ always converges to the double ray on $\mathfrak{I}$ given by $y=$ $0, x \geqq 0$, as the distinct points $t, u, v, w$ converge on $A$ to $p$ in any manner. Thus $A$ satisfies AIII' at $p$.

Lemma 6. AIII' and AII imply AIII and

$$
\pi\left(p^{4}\right)=\lim _{w \rightarrow p} \pi\left(p^{3}, w\right)=\lim _{t, u, v, w \rightarrow p} \pi(t, u, v, w) .
$$

Proof. Any accumulation parabola of the $\pi\left(p^{3}, w\right)$ as $w$ tends to $p$, can be approximated in turn by a $\pi\left(p^{2}, v, w\right)$, a $\pi(p, u, v, w)$ and a $\pi(t, u, v, w)$.

\subsection{We wish to prove}

Theorem 3. Let A satisfy $\mathrm{AIII}^{\prime}$ at $p$. Then A will satisfy $\mathrm{AI}^{\prime}$ at $p$. It will also satisfy $\mathrm{AII}^{\prime}$ at $p$ if, in addition to AIII', any of the following conditions holds. on $\mathfrak{T}$.

(i ) $\pi=\lim _{t, u, v, w \rightarrow p} \pi(t, u, v, w)$ is nondegenerate or the double line

(ii) $p$ is an end-point of $A$,

(iii) A satisfies AII at $p$.

Proof. Assume that $A$ satisfies $\mathrm{AIII}^{\prime}$ at $p$. Since $\pi(t, u, v, w)$ exists when $t, u, v, w$ are close to $p$, we may assume that $A$ is convex.

(i) Let $\pi$ be nondegenerate. Then the tangent of $\pi$ at $p$ will be the tangent $\mathfrak{I}$ of $A$ at $p$; cf; [1, 2.12]. Thus $A$ satisfies $\mathrm{AI}^{\prime}$ at $p$. Using the convexity of both $A$ and $\pi$, we readily verify that $A-\{p\}$ and $\pi-\{p\}$ will lie on the same side of $\mathfrak{T}$.

Let $R$ be a point on $\pi, R \neq p$. Let $Q \in \pi(t, u, v, w)$ and let $Q$ converge to $R$ as $t, u, v, w$ tend to $p$. Then $\pi(t, u, v, w)$ is one of the $\pi_{i}(t, u, v, Q)$, say $\pi(t, u, v, Q)$, and $\lim _{t, u, v \rightarrow p Q \rightarrow R} \pi(t, u, v, Q)=\pi$. Since $A$ is convex, and $A$ and $R$ lie on the same side of $\mathfrak{T}, \pi_{i}(t, u, v, R)$ will exist when $t, u, v$ are sufficiently close to $p$. Any accumulation parabola $\pi_{i}$ of the $\pi_{i}(t, u, v, R)$ will meet $\pi$ at $p$ with a multiplicity $\geqq 3$, and will also pass through $R$. Hence either $\pi_{1}$ or $\pi_{2}$ will coincide with $\pi$; cf. [2, 3.3]. Thus $A$ satisfies $\mathrm{AII}^{\prime}$ at $p$ if $\pi$ is nondegenerate. We shall deal with the case where $\pi$ is the double line on $\mathfrak{I}$ after considering case (ii).

(ii) Let $p$ be an end-point of $A$. By Lemma $1, A$ satisfies $\mathrm{AI}^{\prime}$ and $\mathrm{AI}$ at $p$. Again $\mathfrak{T}$ denotes the tangent of $A$ at $p$.

Let $R$ be a point which lies on the same side of $\mathfrak{I}$ as $A-\{p\}$ and let $R \notin \pi$. Then there exists a neighborhood $B$ of $p$ on $A$ such that each of the parabolas $\pi_{i}(t, u v, R)$ does not meet $B$ elsewhere. Let $\pi\left(p^{2}, t, R\right)[\pi(p, u, v, R)]$ denote one of the $\pi_{i}\left(p^{2}, t, R\right)\left[\pi_{i}(p, u, v, R)\right]$, $i=1,2$. As a limiting case, as $t$ moves continuously and monotoni- 
cally on $B, \pi\left(p^{2}, t, R\right)$ moves continuously and monotonically in one of the families of tangent parabolas of $B$ at $p$ through $R$. Hence $\lim _{t \rightarrow p} \pi\left(p^{2}, t, R\right)$ exists. Thus $A$ satisfies AII at $p$.

Let $p, u, v, w$ lie on $B$ in that order. Then $\pi(p, u, v, R)$ lies between $\pi\left(p^{2}, v, R\right)$ and $\pi(p, v, w, R)$ in one of the families of parabolas through $p, v$, and $R$, for each choice of $u$ on $B$ between $p$ and $w$. Letting $u$ and $v$ converge to $p$, one obtains that any accumulation parabola of the $\pi(p, u, v, R)$ lies between $\pi\left(p^{3}, R\right)$ and $\pi\left(p^{2}, w, R\right)$ for each choice of $w$ on $B$. Letting $w$ tend to $p$, we obtain that $\lim _{u, v \rightarrow p} \pi(p, u, v, R)=$ $\pi\left(p^{3}, R\right)$.

By using a similar argument, one now readily verifies that

$$
\lim _{t, u, v \rightarrow p} \pi(t, u, v, R)=\pi\left(p^{3}, R\right) .
$$

Thus, $A$ satisfies AII' in case (ii) also.

Next, we deal with the case where $p$ is an interior point of $A$ and $\pi$ is degenerate. From the above, each of the subares into which $A$ is decomposed by $p$ will satisfy AII and AIII' at $p$. By Lemma 6 AIII. By the same Lemma $\pi=\pi\left(p^{4}\right)$ with respect to each of these subarcs. It follows that $\pi$ will have to be a double ray (Types $1 \mathrm{~b}$, 1c or 2), or a double line (Type 3), on each of the one-sided tangents of $A$ at $p$. Hence, these one-sided tangents coincide and $A$ satisfies A I at $p$. Since $A$ is convex, however, it also satisfies $\mathrm{AI}^{\prime}$ at $p$. Thus AIII' always implies $\mathrm{AI}^{\prime}$.

From the above, we observe that if $\pi$ is the double line on $\mathfrak{I}$, then the one-sided osculating parabolas will be pairs of parallel lines one of which is $T$ and AII will be satisfied automatically at $p$. This reduces our discussion to the case (iii).

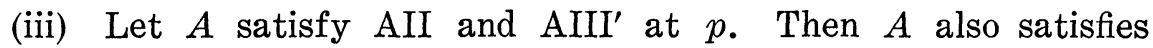
AI and AIII there. Thus the families $\tau$ and $\sigma$ of tangent and osculating parabolas of $A$ at $p$ are defined and so is the superosculating parabola $\pi\left(p^{4}\right)$. Let $R$ be a point on the same side of $\mathfrak{I}$ as $A$ $\{p\} ; R \notin \pi\left(p^{4}\right)$. We may assume that $p$ is an interior point of $A$. Put $A=B \cup p \cup B^{\prime}$. It is sufficient to prove that

$$
\lim _{\substack{t, u, v \rightarrow p \\ t \in B^{\prime}, u, v \in B}} \pi(t, u, v, R)=\pi\left(p^{3}, R\right) \text {. }
$$

where $\pi(t, u, v, R)$ is one of the $\pi_{i}(t, u, v, R)$.

The proof is similar to that given in (ii). There exists a neighborhood $M=N \cup p \cup N^{\prime}$ of $p$ on $A$ such that the parabola $\pi(t, u, v, R)$ does not meet $A$ elsewhere if $t, u, v$ lie on $M$. Let $s, t \in N^{\prime} ; u, v \in N$. Then $\pi(t, u, v, R)$ will lie between $\pi(s, u, v, R)$ and $\pi(p, u, v, R)$ in one of the families of parabolas through $u, v, R$ for each choice of $t$ between $p$ and $s$ on $N^{\prime}$. Letting $t, u, v$ tend to $p$, we obtain that 
any accumulation parabola of the $\pi(t, u, v, R)$ will lie between $\pi\left(s, p^{2}, R\right)$ and $\pi\left(p^{3}, R\right)$ for each choice of $s$ on $N^{\prime}$. Letting $s$ tend to $p$, we obtain that $\lim \pi(t, u, w, R)=\pi\left(p^{3}, R\right)$.

5.6. The method used to prove parts (ii) and (iii) of Theorem 3 can be generalized and will be needed later on.

Let $A$ be a plane arc of order $n$ with respect to an $n$-parameter family of characteristic curves $K$. Let $p, t_{1}, \cdots, t_{n}, t_{n+1}$ be distinct points of $A$ in that order and let $K\left(t_{1}, \cdots, t_{n}\right)$ denote the unique curve of $K$ determined by $t_{1}, \cdots, t_{n}$. Assume that

$$
K\left(p^{n-1}, t_{n}\right)=\lim _{t_{1}, \cdots, t_{n-1} \rightarrow p} K\left(t_{1}, \cdots, t_{n-1}, t_{n}\right)
$$

exists and

$$
K\left(p^{n}\right)=\lim _{t_{n} \rightarrow p} K\left(p^{n-1}, t_{n}\right)=\lim _{t_{2}, \cdots, t_{n} \rightarrow p} K\left(p, t_{2}, \cdots, t_{n}\right)
$$

exists and is nondegenerate.

We wish to show that

$$
\lim _{t_{1}, \cdots, t_{n} \rightarrow p} K\left(K t_{1}, \cdots, t_{n}\right)=K\left(p^{n}\right) .
$$

Proof. $K\left(t_{1}, \cdots, t_{n}\right)$ will 'lie between' $K\left(p, t_{2}, \cdots, t_{n}\right)$ and $K\left(t_{2}, \cdots\right.$, $\left.t_{n}, t_{n+1}\right)$ in the one-parameter subfamily of $K$ through the points $t_{2}, \cdots, t_{n}$. Hence if $t_{1}, \cdots, t_{n}$ tend to $p$, any accumulation curve of the $K\left(t_{1}, \cdots, t_{n}\right)$ will lie between $K\left(p^{n}\right)$ and $K\left(p^{n-1}, t_{n+1}\right)$ for each choice of $t_{n+1}$ on $A$. Letting $t_{n-1}$ tend to $p$ we obtain the desired result.

\section{Strong conical differentiability.}

6.1. Let $A$ be an arc in the real projective plane. If $A$ satisfies PI then $\tau$ will denote the family of nondegenerate tangent conics of $A$ at $p$. The closure $\bar{\tau}$ of the tangent conics is discussed in [3, 3.1].

Condition PII. Let $A$ satisfy PI at $p$ and let $Q$ and $R$ be any fixed points, $Q \notin \mathfrak{T}, R \notin \mathfrak{I} ; p, Q, R$ not collinear. If $s$ is close to $p$, $s \neq p$, the unique tangent conic $\gamma(\bar{\tau}, s, Q, R)$ or $\gamma\left(p^{2}, s, Q, R\right)$ of $A$ at $p$ through $Q, R$ and $s$ converges as $s$ tends to $p$; cf. [3, 5.1].

The limiting osculating conic of $A$ at $p$ through $Q$ and $R$ is denoted by $\gamma(\sigma ; Q, R)$ or $\gamma\left(p^{3}, Q, R\right)$. The family of all the osculating conics of $A$ at $p$ is denoted by $\sigma$.

If PII holds, then $\sigma$ is one of the following three subsets of $\bar{\tau}$ :

Type 1. $\sigma$ consists of all the conics of $\tau$ which have at least three-point contact at $p$ with any particular member of $\sigma$;

Type 2. $\sigma$ consists of the pairs of distinct lines through $p$, both 
of them different from $\mathfrak{T}$;

Type 3. $\sigma$ consists of the pairs of lines one of which is $\mathfrak{I}$ while the other does not pass through $p$; cf. [3, Th. 5].

Any accumulation line of the lines $\mathcal{L}(u, v)$ as $u$ and $v$ tend to $p$ on $A$ is called a general tangent of $A$ at $p$.

Condition PII'. There exist two distinct points $Q$ and $R$, which are not collinear with $p$ and which do not lie on a general tangent of $A$ at $p$, with the following properties. If $t, u, v$ are mutually distinct and lie sufficiently close to $p$ on $A$, then the conic $\gamma(t, u, v, Q, R)$ exists. It converges as $t, u, v$ converge to $p$.

We note that the above condition is weaker than the corresponding condition in [4, 3.2], which also assumes PI.

Condition PII' does not imply $\mathrm{PI}$ or $\mathrm{PI}^{\prime}$ at $p$. The example used in $\S 4$ also satisfies $\mathrm{PII}^{\prime}$ at $p$ and

$$
\lim _{t, u, v \rightarrow p} \gamma(t, u, v, Q, R)=\mathfrak{L}(p, Q) \cup \mathfrak{L}(p, R) .
$$

This arc, however, does not satisfy Condition PI.

We shall prove some of the assertions made in $[4,3.2]$ in the following.

Theorem 4. Let $A$ satisfy $\mathrm{PII}^{\prime}$ at $p$. Then A will satisfy $\mathrm{PI}^{\prime}$ if any one of the following also holds.

(i) $\gamma=\lim _{t, u, v \rightarrow p} \gamma(t, u, v, Q, R)$ is not a pair of lines through $p$,

(ii) $p$ is an end-point of $A$,

(iii) A satisfies PI at $p$.

Proof. Let $A$ satisfy $\mathrm{PII}^{\prime}$ at $p$.

(i) Assume that $\gamma$ is nondegenerate or a pair of lines one of which $\mathfrak{L}(Q, R)$. Then $\gamma$ has a unique tangent $\mathfrak{I}$ at $p$. Thus $\mathfrak{I}=\lim \mathfrak{L}(U, V)$ where $U$ and $V$ lie on $\gamma(t, u, v, Q, R)$ and $U$ and $V$ tend to $p$ as $t, u, v$ tend to $p$. In particular, $\mathfrak{I}=\lim _{u, v \rightarrow p} \mathfrak{L}(u, v)$. Thus $A$ satisfies $\mathrm{PI}^{\prime}$ at $p$.

(ii) Let $p$ be an end-point of $A$. Choose a point $S$ such that no three of $p, Q, R, S$ are collinear and $S$ does not lie on $\gamma$. Then there exists a neighborhood $B$ of $p$ on $A$ such that no conic through $Q, R$ and $S$ will meet $B$ more than twice. It follows that $\gamma(p, t, Q, R, S)$ varies continuously and monotonically in the pencil of conics through $p, Q, R, S$ as $t$ moves continuously and monotonically on $B$ to $p$. Hence $\gamma_{0}=\lim _{t \rightarrow p} \gamma(p, t, Q, R, S)$ exists. Now $\gamma_{0}$ is not a pair of lines through $p$, and hence $\gamma_{0}$ has a tangent $\mathfrak{I}$ at $p$. As in (i), $\lim _{t \rightarrow p} \mathfrak{L}(p, t)=\mathfrak{T}$. Thus $A$ satisfies PI at $p$. It will be shown in (iii) below that PI and $\mathrm{PII}^{\prime}$ imply $\mathrm{PI}^{\prime}$. 
(iii) Assume that $A$ satisfies $\mathrm{PI}$ and $\mathrm{PII}^{\prime}$ at $p$. Then $A$ also satisfies PII there. Let $\mathfrak{I}$ be the tangent of $A$ at $p$. Choose points $Q, R$ such that $p, Q, R$ are not collinear and $Q$ and $R$ do not lie on $\mathfrak{T}$. Then $\gamma\left(p^{3}, Q, R\right)$ exists.

Next, choose $S \notin \gamma\left(p^{3} Q, R\right) \cup \mathfrak{T}$ and such that no three of the points $p, Q, R, S$ are collinear. Then there exists a neighborhood $B$ of $p$ on $A$ such that no conic through $Q, R$ and $S$ meets $B$ more than twice.

Let $t, u, p, v$ lie on $B$ with $u$ between $p$ and $t$. Then $\gamma(u, v, Q, R, S)$ will lie between $\gamma(t, v, Q, R, S)$ and $\gamma(p, v, Q, R, S)$ in the family of conics through $v, Q, R, S$. By applying $\S 5.6$ we obtain that $\lim _{u, v \rightarrow p} \gamma(u, v, Q, R, S)=\gamma\left(p^{2}, Q, R, S\right)$. By our choice of $S, \gamma\left(p^{2}, Q, R, S\right)$ is nondegenerate. Hence it has a tangent at $p$ and this tangent is I. Hence $\lim _{u, v \rightarrow p} \mathfrak{R}(u, v)=\mathfrak{I}$ as before.

\subsection{The next condition is:}

Condition PIII. $A$ satisfies PII at $p$ and if $Q \notin \mathfrak{T}$, then $\gamma\left(p^{3}, s, Q\right)$ converges as $s$ tends to $p$ on $A$.

The limit superosculating conic of $A$ at $p$ through $Q$ is denoted by $\gamma\left(p^{4}, Q\right)$. If $p$ is Type 1 , then the family $\rho$ of all the superosculating conics of $A$ at $p$ is one of the following subsets of $\sigma$.

Type 1a. $\rho$ is a subfamily of $\sigma$ consisting of all those conics of $\sigma$ which have four-point contact at $p$ with a particular conic of $\sigma$.

Type 1b. $\rho$ consists of all pairs of lines through $p$, one of which is $\mathfrak{I} ;$ cf. $[3,6]$.

In Types 2 and 3, PIII is satisfied automatically.

Condition PIII'. There exists a point $R$, which does not lie on a general tangent of $A$ at $p$, with to following properties. If $t, u, v, w$ are mutually distinct and lie sufficiently close to $p$, the conic $\gamma(t, u, v, w, R)$ is uniquely defined. It converges as $t, u, v, w$ converge to $p$.

The above condition is weaker than that in $[4,3.4]$ which assumes PII'.

Remark. If $A$ satisfies PIII' at $p$ and $\lim \gamma(t, u, v, w, R)=\gamma$, then $\lim _{Q \rightarrow R} \gamma(t, u, v, w, Q)=\gamma$; for any accumulation conic of the $\gamma(t, u, v, w, Q)$ will have four-point contact with $\gamma$ at $p$ and it will also pass through $R$. Similarly, if $S \neq p$ and there is a sequence of points $Q$ tending to $S$ such that $\gamma(t, u, v, w, Q)$ converges, then $\gamma(t, u, v, w, S)$ converges to the same limit; thus PIII' holds. 
We observe that PIII' does not imply $\mathrm{PII}^{\prime}$ or even PII, as the example $A=A_{4} \cup p \cup A_{4}^{\prime}$, in $\S 5.4$ shows. The arc $A$ satisfies PIII' at $p$. In particular, if $R$ does not lie on the $x$-axis, then $\lim _{t, u, v, w \rightarrow p} \gamma(t, u, w, R)=$ $\mathfrak{L}(p, R) \cup \mathfrak{I}$. The osculating conics of $A_{4}$ and of $A_{4}^{\prime}$ at $p$ are given by

$$
a\left(x^{2}-y\right)+b x y+c y^{2}=0 \text { and } a\left(x^{2}-y / 4\right)+b x y+c y^{2}=0,
$$

respectively. Hence $A$ does not satisfy PII at $p$.

We wish to prove

Theorem 5. Let $A$ satisfy $\mathrm{PIII}^{\prime}$ at $p$. Then $A$ will satisfy $\mathrm{PI}^{\prime}$ at $p$. It will also satisfy $\mathrm{PII}^{\prime}$ at $p$ if, in addition, any one of the following holds.

(i) $\gamma=\lim _{t, u, v, w \rightarrow p} \gamma(t, u, v, w, R)$ is nondegenerate,

(ii) $p$ is an end-point of $A$,

(iii) A satisfies PII at $p$.

Proof. Let $A$ satisfy PIII' at $p$.

(i) Assume that $\gamma$ is nondegenerate. Let $Q \in \gamma, Q \neq p, Q \neq R$. Then any accumulation conic $\gamma_{0}$ of the $\gamma(t, u, v, Q, R)$ will have threepoint contact with $\gamma$ at $p$ and will pass through $Q$ and $R$. Hence $\gamma=\gamma_{0}$. Thus $A$ satisfies $\mathrm{PII}^{\prime}$ at $p$. Since

$$
\lim _{t, u, v \rightarrow p} \gamma(t, u, v, Q, R)=\gamma
$$

is nondegenerate, $A$ also satisfies $\mathrm{PI}, \mathrm{PI}^{\prime}$ and $\mathrm{PII}$ at $p$.

(ii) Let $p$ be an end-point of $A$. Choose a point $Q$ not on a general tangent of $A$ at $p$ such that $Q \notin \gamma$ and $p, Q, R$ are not collinear. Then there exists a neighborhood $B$ of $p$ on $A$ such that no conic through $Q$ and $R$ meets $B$ more than three times. By applying $\S 5.6$ one verifies that $B$ satisfies $\mathrm{PI}$ and PII at $p$. Then using the technique in $[4,5.2]$, one shows that $A$ satisfies $\mathrm{PII}^{\prime}$ at $p$. By $\S 6.1, A$ also satisfies $\mathrm{PI}^{\prime}$ there.

We now verify that $\mathrm{PIII}^{\prime}$ implies $\mathrm{PI}^{\prime}$ when $\gamma$ is degenerate and $p$ is an interior point of $A$. Let $A=B \cup p \cup B^{\prime}$. Then $B$ and $B^{\prime}$ both satisfy $\mathrm{PIII}^{\prime}$ at $p$. By (ii), they also satisfy $\mathrm{PI}^{\prime}$ and $\mathrm{PII}^{\prime}$. Let $\mathfrak{I}$ and $\mathfrak{I}^{\prime}$ be the tangents of $B$ and $B^{\prime}$ respectively at $p$.

Since $\gamma$ is degenerate, it must be $\mathfrak{I} \cup \mathfrak{L}(p, R)$ when associated with $B$ and $\mathfrak{I}^{\prime} \cup \mathfrak{L}(p, R)$ when associated with $B^{\prime}$. Hence $\mathfrak{I}=\mathfrak{I}^{\prime}$. Thus $A$ satisfies PI at $p$.

Since $\gamma(t, u, v, w, R)$ always converges as $t, u, v, w$ converge on $A$ to $p$, we readily verify that $A$ has no cusp at $p$. By Lemma $3, A$ satisfies $\mathrm{PI}^{\prime}$ at $p$.

(iii) Assume that $A$ satisfies PII and PIII' at $p$. Then $A$ satisfies PIII there. From the above, $A=B \cup p \cup B^{\prime}$ also satisfies $\mathrm{PI}^{\prime}$ at $p$ and 
both $B$ and $B^{\prime}$ will satisfy $\mathrm{PII}^{\prime}$ there. Let $\mathfrak{I}$ be the tangent of $A$ at $p$. Let $Q$ and $R$ be points such that $Q \notin \mathfrak{I}, R \notin \mathfrak{I} ; p, Q, R$ are not collinear, and $Q \in \gamma\left(p^{4}, R\right)$.

We wish to prove that $\gamma(t, u, v, Q, R)$ tends to $\gamma\left(p^{3}, Q, R\right)$ if $t, u, v$ tend to $p$. We may assume that $t, p, u, v$ lie on $A$ in that order with $t$ on $B^{\prime}$ and $u, v$ on $B$.

Since PIII' holds, a conic through $R$ and four points of $A$ tends to $\gamma\left(p^{4}, R\right)$ as these four points tend to $p$ on $A$. Hence there exists a neighborhood $M$ of $p$ on $A$ such that no conic through $Q$ and $R$ meets $M$ more than three times. The proof then follows the method of $\S 5.6$.

\subsection{Conical conditions IV and IV'.}

Condition PIV. The arc $A$ satisfies PIII and the superosculating conic $\gamma\left(p^{4}, s\right)$ converges as $s$ tends to $p$.

The limit ultraosculating conic of $A$ at $p$ is denoted by $\gamma\left(p^{5}\right)$. It is nondegenerate (Type 1a(i)), or the point conic $p$ (Type 1a(ii)), or the double line on $\mathfrak{T}$ (Type 1a(iii)); cf. [3, 7].

In the remaining cases, Types $1 \mathrm{~b}, 2$ and 3 , PIV is satisfied automatically and $\gamma\left(p^{5}\right)$ is the double line on $\mathfrak{T}$.

Condition $\mathrm{PIV}^{\prime} . \quad \gamma(s, t, u, v, w)$ is uniquely defined and converges as the mutually distinct points $s, t, u, v, w$ converge on $A$ to $p$.

The above condition is weaker than the corresponding one in [4, 3.6] which also assumes PIII'.

We note that PIV' does not imply PIII'. To show this, let $A=$ $A_{5}^{\prime} \cup p \cup A_{5}$, where $p$ is the origin, $A_{5}$ is given by $x=s^{2}, y=$ $s^{4}+s^{7}, 0<s<\delta$; and $A_{5}^{\prime}$ is given by $x=-s^{2}-s^{4}, y=s^{4}+s^{7}, 0<s<\delta ; \delta$ is sufficiently small. Here $p$ is of Type 1a(ii) with respect to both $A_{5}$ and $A_{5}^{\prime}$. It can be verified that $A$ satisfies PIV $^{\prime}$ at $p$. The families of superosculating conics of $A_{5}$ and $A_{5}^{\prime}$ are distinct, however, and hence PIII and PIII' do not hold there.

A similar counterexample exists for Type 1a(iii).

We wish prove

Theorem 6. Let A satisfy $\mathrm{PIV}^{\prime}$ at $p$. Then $A$ will satisfy $\mathrm{PI}^{\prime}$ and $\mathrm{PII}$ there. A will also satisfy $\mathrm{PIII}^{\prime}$ at $p$ if any one of the following holds.

(i) $\lim \gamma(s, t, u, v, w)$ is nondegenerate.

(ii) $p$ is an end-point of $A$.

(iii) A satisfies PIII at $p$.

Proof. Let $A$ satisfy $\mathrm{PIV}^{\prime}$ at $p$

(i) Assume that $\gamma$ is nondegenerate. Let $R \in \gamma, R \neq p$, and let 
$Q \in \gamma(s, t, u, v, w), Q \rightarrow R$. Then $\lim \gamma(t, u, v, w, Q)=\lim \gamma(s, t, u, v, w)=$ $\gamma$. By the Remark in $\S 6.2, A$ satisfies PIII' at $p$. By Theorem $5, A$ also satisfies $\mathrm{PII}^{\prime}$ and $\mathrm{PI}^{\prime}$ at $p$.

(ii) Let $p$ be an end-point of $A$. Choose a point $R \notin \gamma$. Then there exists a neighborhood $B$ of $p$ on $A$ such that no conic through $R$ meets $B$ more than four times. By applying $\S 5.6$, one can show that $B$ satisfies $\mathrm{PI}, \mathrm{PI}^{\prime}$. We may assume $R \notin \mathfrak{T}$ and again apply 5.6 to obtain PII and PIII at $p$. Then using the technique in $[4, \S 5]$, one

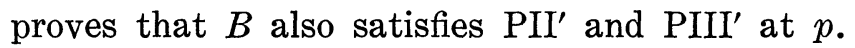

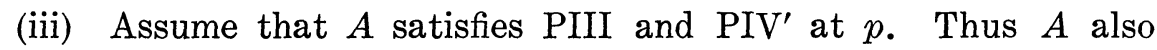
satisfies PI, PII and PIV there. Thus $\gamma=\gamma\left(p^{5}\right)$.

We may assume that $p$ is an interior point of $A$; thus $A=$ $B \cup p \cup B^{\prime}$, say. By (ii), $B$ and $B^{\prime}$ will satisfy $\mathrm{PI}^{\prime}, \mathrm{PII}^{\prime}$ and $\mathrm{PIII}^{\prime}$ at $p$.

Let $R \notin \mathfrak{I} \cup \gamma\left(p^{5}\right)$. Following $\S 5.6$ we can show that $\gamma\left(t^{\prime}, t, u, v, R\right)$ and $\gamma\left(u^{\prime}, t^{\prime}, t, u, R\right)$ both tend to $\gamma\left(p^{4}, R\right)$ as $t, u, v$ tend to $p$ on $B$ and $t^{\prime}, u^{\prime}$ tend to $p$ on $B^{\prime}$. The details will be omitted. This proves PIII'.

Next, we show that $A$ satisfies $\mathrm{PI}^{\prime}$ at $p$, assuming only PIV'. From now on we may take $\gamma=\lim \gamma(s, t, u, v, w)$ to be degenerate and $p$ to be an interior point of $A$. If $A=B \cup p \cup B^{\prime}$, then both $B$ and $B^{\prime}$ will satisfy PIV at $p$. Hence $\gamma=\gamma\left(p^{5}\right)$ is the point $p$ or a double line. There exists a neighborhood $M=N \cup p \cup N^{\prime}$ of $p$ on $A$ which is convex; otherwise a double segment, for example, would be among the accumulation conics through five points of $A$.

Case 1. $\gamma\left(p^{5}\right)$ is a double line. Then $\gamma\left(p^{5}\right)$ lies on both of the one-sided tangents $\mathfrak{I}$ and $\mathfrak{I}^{\prime}$ of $A$ at $p$. Hence these tangents coincide. By Lemma $2, A$ will satisfy $\mathrm{PI}^{\prime}$ at $p$.

Case 2. $\gamma\left(p^{5}\right)=p$; thus, $p$ is of Type 1a(ii) with respect to both $B$ and $B^{\prime}$. Since $M$ is convex, $M$ will not cross $\mathfrak{I}$ or $\mathfrak{I}^{\prime}$ at $p$. Suppose that $\mathfrak{I} \neq \mathfrak{I}^{\prime}$. Choose a point $R \notin \mathfrak{I} \cup \mathfrak{I}^{\prime}$ such that $R$ and $M-\{p\}$ lie in the same region bounded by $\mathfrak{T}$ and $\mathfrak{T}^{\prime}$. If $t^{\prime} \in N^{\prime}$, then the $\gamma\left(p^{3}, t^{\prime}, R\right)$ of $B$ do not meet $M$ elsewhere. It is known that $\gamma\left(p^{5}, t^{\prime}, R\right)$ and $B$ touch $\mathfrak{T}$ on the same side of $\mathfrak{T}$. Hence the arc of $N^{\prime}$ between $p$ and $t^{\prime}$ will lie inside $\gamma\left(p^{3}, t^{\prime}, R\right)$; cf. [3, 3.3]. It follows that as $t^{\prime}$ tends to $p, \gamma\left(p^{3}, t^{\prime}, R\right)$ will tend to the pair of lines $\mathscr{L}(p, R) \cup \mathfrak{I}$, which intersects $A$ at $p$; cf. [3,5 .11]. Hence the end-points of $M$ will lie on opposite sides of $\gamma\left(p^{3}, t^{\prime}, R\right)$ if $t^{\prime}$ is sufficiently close to $p$ and $\gamma\left(p^{3}, t^{\prime}, R\right)$ will meet $M$ with an odd multiplicity. Since $M$ already meets $\gamma\left(p^{3}, t^{\prime}, R\right)$ four times, it will meet $M$ at least five times. This is a contradiction. It follows that $\mathfrak{I}=\mathfrak{I}^{\prime}$ and by Lemma $2, A$ satisfies $\mathrm{PI}^{\prime}$ at $p$. Finally, we show that PIV' implies PII'. Let $R$ be a point, $R \notin \mathfrak{I} \cup \gamma$ and again assume that no conic through $R$ meets a suitable neighborhood $M_{1}$ of $p$ on $A$ more than four times. Let $d \in M_{1}$ and choose a 
neighborhood $M$ of $p$ on $M_{1}$ such that $d \notin M$. Then no conic through $R$ and $d$ will meet $M$ more than three times. We may also assume that $\mathfrak{L}(p, R) \cup \mathfrak{I}$ does not meet $M$ outside $p$. The proof then follows the method of $[4,6.3]$.

The authors wish to thank Peter Scherk for reading the manuscript and making many valuable suggestions.

\section{REFERENCES}

1. N. D. Lane, Parabolic differentiation, Canad. J. Math. 15 (1963), 546-162.

2. $\quad$ Arcs of parabolic order four, Canad. J. Math. 16 (1964), 321-338.

3. N. D. Lane and K. D. Singh, Conical differentiation, Canad. J. Math. 16 (1964), 169-190.

4. N. D. Lane and K. D. Singh, Arcs of conical order five, J. Reine Angew. Math. 217 (1965), 109-127.

5. N. D. Lane and K. D. Singh, Order and characteristic of parabolically differentiable points, Ann. Mat. Pura Appl. 71 (1966), 127-164.

6. N. D. Lane and K. D. Singh, Characteristic and order of conically differentiable points, J. Reine Angew. Math. 224 (1966), 164-684.

7. N. D. Lane and Peter Scherk, Differentiable points in the conformal plane, Canad. J. Math. 5 (1953), 512-518.

8. N. D. Lane and Peter Scherk, Characteristic and order of differentiable points in the conformal plane, Trans. Amer. Math. Soc. 81 (1956), 358-378.

9. O. Haupt, G. Aumann and C. Pauc, Differential und Integralrechnung, II, Berlin, (1950).

10. T. Popoviciu, Les fonctions convexes, Paris (1945).

11. O. Haupt, H. Künneth, Geometrische Ordnungen, Berlin (1967).

Received November 28, 1967.

MCMASTER UNiVersity, UNiversity of California, Los ANGeles, AND

LUCKNOW UNIVERSITY 



\section{PACIFIC JOURNAL OF MATHEMATICS}

\section{EDITORS}

\author{
H. ROYDEN \\ Stanford University \\ Stanford, California \\ R. R Phelps \\ University of Washington \\ Seattle, Washington 98105
}

\author{
J. Dugundui \\ Department of Mathematics \\ University of Southern California \\ Los Angeles, California 90007
}

RICHARD ARENS

University of California

Los Angeles, California 90024

\section{ASSOCIATE EDITORS}
E. F. BeCKENBACH
B. H. NeUmanN
F. WolF
K. YosidA

\section{SUPPORTING INSTITUTIONS}

\author{
UNIVERSITY OF BRITISH COLUMBIA \\ CALIFORNIA INSTITUTE OF TECHNOLOGY \\ UNIVERSITY OF CALIFORNIA \\ MONTANA STATE UNIVERSITY \\ UNIVERSITY OF NEVADA \\ NEW MEXICO STATE UNIVERSITY \\ OREGON STATE UNIVERSITY \\ UNIVERSITY OF OREGON \\ OSAKA UNIVERSITY \\ UNIVERSITY OF SOUTHERN CALIFORNIA
}

\author{
STANFORD UNIVERSITY \\ UNIVERSITY OF TOKYO \\ UNIVERSITY OF UTAH \\ WASHINGTON STATE UNIVERSITY \\ UNIVERSITY OF WASHINGTON

$* * * * *$
AMERICAN MATHEMATICAL SOCIETY
CHEVRON RESEARCH CORPORATION
TRW SYSTEMS
NAVAL WEAPONS CENTER

The Supporting Institutions listed above contribute to the cost of publication of this Journal, but they are not owners or publishers and have no responsibility for its content or policies.

Mathematical papers intended for publication in the Pacific Journal of Mathematics should be in typed form or offset-reproduced, double spaced with large margins. Underline Greek letters in red, German in green, and script in blue. The first paragraph or two must be capable of being used separately as a synopsis of the entire paper. It should not contain references to the bibliography. Manuscripts, in duplicate if possible, may be sent to any one of the four editors. Please classify according to the scheme of Math. Rev. 36, 1539-1546. All other communications to the editors should be addressed to the managing editor, Richard Arens, University of California, Los Angeles, California, 90024.

50 reprints are provided free for each article; additional copies may be obtained at cost in multiples of 50 .

The Pacific Journal of Mathematics is published monthly. Effective with Volume 16 the price per volume (3 numbers) is $\$ 8.00$; single issues, $\$ 3.00$. Special price for current issues to individual faculty members of supporting institutions and to individual members of the American Mathematical Society: $\$ 4.00$ per volume; single issues $\$ 1.50$. Back numbers are available.

Subscriptions, orders for back numbers, and changes of address should be sent to Pacific Journal of Mathematics, 103 Highland Boulevard, Berkeley, California, 94708.

PUBLISHED BY PACIFIC JOURNAL OF MATHEMATICS, A NON-PROFIT CORPORATION

Printed at Kokusai Bunken Insatsusha (International Academic Printing Co., Ltd.), 7-17. Fujimi 2-chome, Chiyoda-ku, Tokyo, Japan. 


\section{Pacific Journal of Mathematics}

Vol. 28, No. 3

May, 1969

Jon F. Carlson, Automorphisms of groups of similitudes over $F_{3} \ldots \ldots \ldots$

W. Wistar (William) Comfort, Neil Hindman and Stelios A. Negrepontis,

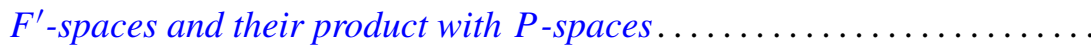

Archie Gail Gibson, Triples of operator-valued functions related to the unit

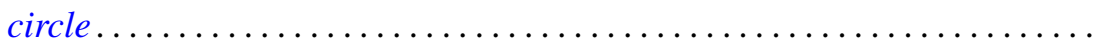

David Saul Gillman, Free curves in $E^{3}$

E. A. Heard and James Howard Wells, An interpolation problem for

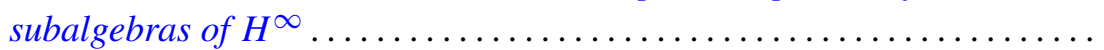

Albert Emerson Hurd, A uniqueness theorem for weak solutions of symmetric

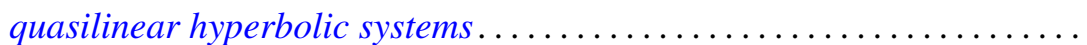

E. W. Johnson and J. P. Lediaev, Representable distributive Noether

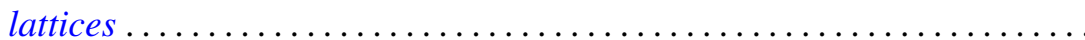

David G. Kendall, Incidence matrices, interval graphs and seriation in

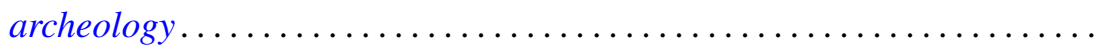
565

Robert Leroy Kruse, On the join of subnormal elements in a lattice ....... 571

D. B. Lahiri, Some restricted partition functions; Congruences modulo 3 .... 575

Norman D. Lane and Kamla Devi Singh, Strong cyclic, parabolic and conical differentiability........................................

William Franklin Lucas, Games with unique solutions that are

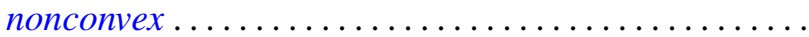

Eugene A. Maier, Representation of real numbers by generalized geometric series.

Daniel Paul Maki, A note on recursively defined orthogonal polynomials ...

Mark Mandelker, $F^{\prime}$-spaces and z-embedded subspaces ...

James R. McLaughlin and Justin Jesse Price, Comparison of Haar series with gaps with trigonometric series

Ernest A. Michael and A. H. Stone, Quotients of the space of irrationals ....

William H. Mills and Neal Zierler, On a conjecture of Golomb ...

J. N. Pandey, An extension of Haimo's form of Hankel convolutions ...

Terence John Reed, On the boundary correspondence of quasiconformal mappings of domains bounded by quasicircles...

Haskell Paul Rosenthal, A characterization of the linear sets satisfying Herz's criterion.

George Thomas Sallee, The maximal set of constant width in a lattice...

I. H. Sheth, On normaloid operators

James D. Stasheff, Torsion in BBSO ...

Billy Joe Thorne, A - P congruences on Baer semigroups.

Robert Breckenridge Warfield, Jr., Purity and algebraic compactness for

modules... 